\title{
Pressure Distribution Management System in 3D Printed Transtibial Prosthetic Socket during Static Loading: A Preliminary Assessment during Stance Phase
}

\author{
Murad Abdullah Subih ( $\nabla$ murad.sabeeh@gmail.com ) \\ Universiti Malaya https://orcid.org/0000-0001-8624-2876 \\ Nooranida Arifin \\ Universiti Malaya \\ Ebrahim Al-Fakih \\ Imam Abdulrahman Bin Faisal University
}

Research

Keywords: interface pressure, control system, 3D printed socket, prosthetic socket, air bladders

Posted Date: May 18th, 2020

DOI: https://doi.org/10.21203/rs.3.rs-28930/v1

License: (c) (i) This work is licensed under a Creative Commons Attribution 4.0 International License.

Read Full License 


\section{Abstract \\ Background}

A management system within a prosthetic socket for efficient redistribution and interface pressure reduction is highly demanded. This research article developed a new control system for interface pressure in a 3D printed transtibial prosthetic socket. This system aimed to redistribute and reduce the high interface pressure along residual limb regions in a 3D printed socket.

\section{Methods}

A 3D printed socket was built with tiny air bladders that were integrated with the liner. This 3D socket had three air bladders located on the posterior, lateral, and medial sides, and connected to three separate mini pumps and valves for inflation and deflation. The air bladders' inflation and deflation were controlled by using a pre-programed keypad. The keypad with an electronic system (electronic circuit, mini pumps, and valves) was attached to a prosthetic pylon in a small box. A universal tensile machine was employed to apply loads into the prosthetic device to resemble human body weight. The interface pressure in the 3D printed socket was evaluated by using F- socket transducers at proximal, middle, and distal regions of an artificial residual limb during inflation and deflation of the air bladders in stance phase.

\section{Results}

The pressure control system minimized the interface pressures within the 3D printed prosthetic socket during inflation. The posterior and medial sides recorded a maximum reduction in pressure during the inflation of posterior and medial air bladders, respectively. Statistically, the highest significant pressure reduction $(p<0.05)$ was $6.81 \%$, which was recorded at the posterior proximal subregion and $3.44 \%(p>$ $0.05)$ at the medial middle, but it was not significant. There was no statistical change in the mean pressure values at the whole anterior side, but there were significant differences during lateral and medial air bladder inflation and deflation.

\section{Conclusions}

This study is intended to recognize the impact of high interface pressure around the residual limb more deeply. The new pressure distribution management system offered rearrangement with minimized interface pressure over the artificial residual limb subregions.

\section{Introduction}

The World Health Organization (WHO) has estimated that 422 million adults have diabetes in 2014, in contrast to 108 million in 1980. People who had amputation because of diabetes were higher than those 
who had amputation caused by other factors [1].The amputees need an artificial substitute or replacement for the missing body parts in the form of prosthetic devices to restore normal functions of missing body parts and carry out regular motions [2]. The prosthetic device for transtibial amputation consists of three fundamental components which include a socket, pylon, and foot. The socket is the core component of a prosthetic device, and it is a coupling structure between the residual and artificial limb (prosthesis) [3, 4]. The socket transmits mechanical loads from the amputee body into the foot, and vice versa [5]. Thereby, socket fitting is an indicator of user's comfort and satisfaction [6-8].

Prosthetists often customize the transtibial prosthetic socket by hand casting and modifying to fit around the stump[6-8]. This conventional socket manufacturer needs a long-time process to fabricate the socket and highlight bone and muscle areas of the stump. Therefore, prosthetists must have professional skills for the socket fabrication [3, 9]. Currently, the three-dimensional (3D) printed machine and computer-aided manufacturing (CAM) are utilized to develop the 3D printed socket model. Computer-aided design (CAD) is employed to edit and manipulate the socket design easily. Therefore, the 3D printed technique has overcome tedious conventional socket fabrication $[8,10]$. An interface pressure of the 3D printed variableimpedance prosthetic socket was evaluated for a transtibial amputee at Biomechatronics Group, Massachusetts Institute of Technology (MIT) [11]. The pressure was recorded by using F-Socket transducers during the stance phase of the gait cycle. This study observed that the contact pressure at the fibula head region and tibia region was reduced during a single-leg standing in contrast to the conventional socket.

The interface pressure within the prosthetic socket can indicate the user's satisfaction and comfort with the socket.[4]. Indeed, the prosthetic socket must meet the pressure tolerance profile of the residual limb by providing a secure suspension system and superior fitting[12]. Prosthesis suspension systems play a critical role in pressure distribution around the stump. [13].The suspension systems set the stump inside the socket via a single distal pin/lock, suction, lanyard, or magnetic coupling [14, 15]. Silicon liner (pin/ lock) and suction systems are commonly used as suspension systems for transtibial prostheses. A pin/ lock liner offers excellent attachment with the residual limb $[13,16]$. However, this liner could increase the interface pressure at the residual limb distal during a swing phase of gait cycle, which causes pain and skin injuries. The suction suspension system provides an excellent fitting and could minimize a quantity of pistoning within a socket as compared to the pin/lock suspension system [14, 17]. A study survey conducted by Dillingham, Pezzin [18] found that 146 amputees were not satisfied with their suspension system because they suffered from skin injuries and pain at the stump during daily activities.

Pressure in the pin/lock suspension system was evaluated via Fiber Bragg Grating during the stance phase by Al-Fakih, Arifin [19]. In this study, a simulation machine was utilized to apply load and generate ten gait cycles to mimic a human body weight and the amputee's gait cycle. Moreover, an artificial stump was built from silicon material to form the amputee's residual limb. The authors observed that the highest values of peak pressure $(\mathrm{kPa})$ of the artificial stump were recorded at the anterior middle (34.18), posterior proximal (42.25), lateral distal (53.99) and medial distal (56.06) during the stance phase. Therefore, the highest peak pressure among all values was at the medial distal region. 
A new suspension system (HOLO) was established by removing the pin from the silicon liner and replacing it with a loop fastener. A velcro strap (hook) was contacted to the socket wall at the medial and lateral areas. Pressure distribution of the new suspension system (HOLO) was evaluated during a walk of 10 healthy subjects by using the F-Socket transducers (9811E) and was compared with a pin/lock suspension system[20]. The findings of this study concluded that high pressure was measured at the lateral and medial sides during the stance phase of the new suspension system (HOLO). However, the pin/lock liner has high pressure at the distal region of the stump in anterior, posterior, and medial areas during the stance phase of gait.

Pressure in Dermo and Seal-In X5 liner was evaluated during a regular walk by using

F- socket sensors in a study by Ali, Osman [21].The highest value of pressure was recorded at the anterior of the stump in Seal-In X5 liner ( $84.9 \mathrm{kPa})$. On the other hand, the pressure at the anterior for the Dermo liner was $60.2 \mathrm{kPa}[21]$.

Furthermore, Eshraghi, Osman [22] investigated the pressure of a new magnetic suspension system and compared it with the pin/lock and seal-in suspension systems by using four F-socket transducers. In this study, the mean peak pressure at the anterior and posterior sides of the pin/lock system was higher than the anterior and posterior of the magnetic suspension system (89.89 kPa vs. $79.26 \mathrm{kPa}$ (Anterior); 47.22 vs. $26.01 \mathrm{kPa}$ ) (posterior), respectively).

Later, a new air pneumatic suspension system (APSS) was developed at the Applied Biomechanics Laboratory in University of Malaya by Pirouzi, Osman [23]. APSS can manage the change of the residual limb size and attachment. During this study, five subjects used APSS during a normal walk to evaluate the performance and pressure distribution. The study observed that the interface pressure was reduced by using APSS. Results showed that the highest value of pressure was recorded the anterior middle $(56.43 \mathrm{kPa})$ [23]. APSS had the lowest value of pressure among all suspension systems in the anterior middle region, whereas, the APSS still had a costly silicon liner material without pins. APSS has an air bladder that is embedded inside the socket wall as an air cuff in a bladder piece. Therefore, inflation and deflation of an air bladder cannot be controlled on one side of the stump with one air bladder piece, and it can be inflated and deflated together at all sides.

The continuous change of the residual limb size or volume concentrates the pressure at a specific area of the stump[23, 24]. Consequently, the amputees suffer from injury and pain of the residual limb, which results in high-pressure distribution [25]. Issues related to the management of interface pressure which has occurred by the continuous change in the stump size, doffing, and donning of new design solutions remain unemployed despite attempts to address these problems[23]. To overcome the high interface pressure within the prosthetic socket, this study aims to redistribute an interface pressure within the 3D printed transtibial prosthetic socket and reduce the high pressure. This article developed a new pressure management system to control pressure distribution inside the 3D printed socket. The hypothesis is that this system would provide a relief in bony areas and convenient coupling at the stump muscle areas with adequate interface pressure. 


\section{Results}

Pressure distribution measurements were extracted in 12 subregions of the artificial residual limb, such as proximal, middle and distal of the anterior, posterior, lateral, and medial sides during the stance phase (heel strike, foot flat, and toe-off). The mean pressure values are illustrated in Table 1. The whole four main regions of the artificial residual limb pressures are presented in Fig. 1. Pressure was reduced and increased in subregions of the artificial stump by utilizing the pressure management system. Posterior and medial sides recorded a maximum reduction in pressure during the inflation of posterior and medial air bladders, respectively. The highest pressure reduction was $(6.81 \%)$, which was recorded at the posterior proximal, and $3.44 \%$ at the medial middle.

The mean pressure for the four major regions of the artificial residual limb was also taken, as shown in Fig. 4. The mean pressure value for the whole posterior side during the inflation was significant and slightly lower than during deflation for both $50 \mathrm{~kg}$ and $65 \mathrm{~kg}$ loads (for $50 \mathrm{~kg}, \mathrm{P}<0.05$, mean $=$ $39.80 \mathrm{kPa}$ (inflation), $\mathrm{SD}=0.91$, mean $=40.73 \mathrm{kPa}$ (deflation), $\mathrm{SD}=0.48$; for65kg, $\mathrm{p}<.05$, mean $=$ $50.47 \mathrm{kPa}$ (inflation), $\mathrm{SD}=0.835$, mean $=52.94 \mathrm{kPa}$ (deflation), $\mathrm{SD}=0.450$,respectively). Furthermore, the mean pressure during the inflation was significantly higher than deflation for the whole lateral side for 50 and $65 \mathrm{~kg}$ loads $(\mathrm{p}<0.05$, mean $=36.81 \mathrm{kPa}$ (inflation), $\mathrm{SD}=1.13$, mean $=34.36 \mathrm{kPa}$ (deflation), $\mathrm{SD}=$ $0.227 ; \mathrm{p}<0.05$, mean $=46 \mathrm{kPa}$ (inflation), $\mathrm{SD}=1.59$, mean $=42.96 \mathrm{kPa}$ (deflation), $\mathrm{SD}=0.208$, respectively). Moreover, the mean pressure during the inflation was significantly lower than deflation at the medial side for $50 \mathrm{~kg}$ load, $(\mathrm{p}<0.05$, mean $=42.19 \mathrm{kPa}$ (inflation), $\mathrm{SD}=0.799$, mean $=$ $43.01 \mathrm{kPa}$ (deflation), $\mathrm{SD}=0.066)$, but not significantly for $65 \mathrm{~kg}(p>0.05(0.18)$, mean = $37.76 \mathrm{kPa}$ (inflation), $\mathrm{SD}=0.316$, mean $=38.96 \mathrm{kPa}$ (deflation), $\mathrm{SD}=0.044$ ). There was no statistical change in mean pressure values at the whole anterior side, but significant differences for both loads $(\mathrm{p}<$ 0.05 , mean $=51.57 \mathrm{kPa}, \mathrm{SD}=0.108$, mean $=51.57 \mathrm{kPa}, \mathrm{SD}=0.075 ; \mathrm{p}<0.05$, mean $=55.89 \mathrm{kPa}, \mathrm{SD}=0.082$, mean $=55.86, \mathrm{SD}=0.051$, respectively). 
Table 1

Mean pressure during stance phase with inflation and deflation of air bladders

\section{Mean Pressure for $50 \mathrm{~kg}$ Load (kPa)}

Artificial stump

Subregions

Anterior Proximal

Anterior Middle

(0.)

Anterior Distal

Posterior Proximal

Posterior Middle

Posterior Distal

Lateral Proximal

$(0.20$

Lateral Middle

34.45

$(0.13)$

32.99

$(0.20)$

34.45

(0.28)

35.65

(0.23)

36.50

(0.05)

42.25

(0.09)

50.28

(0.10)

Lateral Distal

Medial Proximal

Medial Middle

Medial Distal
Mean Pressure for $65 \mathrm{~kg}$ Load

(kPa)

Deflation Inflation P-Value*

(SD)

(SD)

(SD)

Value*

$42.96 \quad P>0.05$

(0.05)

(0.07)

(0.091)

$P<0.05$

42.94

55.96

$\mathrm{P}<0.05$

62.85

$\mathrm{P}<0.05$

55.90

(0.09)

(0.07)

68.74

$P<0.05$

48.19

$P<0.05$

68.73

(0.13)

(0.18)

$(0.08)$

64.55

$41.82 \quad \mathrm{P}<0.05 \quad 64.61$

64.55

$P<0.05$

(1.94)

(0.19)

(0.73)

$38.91 \quad \mathrm{P}<0.05 \quad 50.88$

46.38

$P<0.05$

(3.6)

(0.57)

(1.5)

36.68

$\mathrm{P}<0.05$

43.35

40.49

$\mathrm{P}<0.05$

(0.96)

(0.63)

(1.14)

34.99

$P<0.05$

53.04

51.78

$\mathrm{P}<0.05$

(0.88)

(0.42)

(0.55)

33.17

$\mathrm{P}<0.05 \quad 38.08$

38.16

$P<0.05$

(0.69)

(0.33)

2.0

42.28

$\mathrm{P}<0.05$

37.77

48.06

$P<0.05$

(0.47)

(3.34)

$36.55 \quad \mathrm{P}<0.05 \quad 37.61$

37.42

$P<0.05$

(0.06)

(0.049)

(0.50)

42.00

$\mathrm{P}<0.05 \quad 40.15$

36.71

$P>0.05$

(0.20[)

(0.095)

(0.47)

(0.78)

$48.03 \quad \mathrm{P}<0.05 \quad 39.13$

39.14

$P>0.05$

(2.3)

(0.068)

(0.713)

(0.379)

* Significant differences between inflation and deflation 


\section{Discussion}

This is the first study which employed a 3D printed socket with tiny air bladders and an electronic management system to redistribute the interface pressure through the inflation and deflation of air bladders. This article explains that interface pressure in 3D printed transtibial prosthetic socket reduces by utilizing the interface pressure control system. This system has reduced the mean peak pressure inside the socket, and user can control the relief of peak pressures and residual limb fitting within the socket.

The findings of this study revealed that the mean pressure at the whole posterior side was significantly decreased during posterior air bladder inflation. The pressure reduction was $0.93 \%$ for the $50 \mathrm{~kg}$ load, whereas the pressure reduction was $2.47 \%$ for $65 \mathrm{~kg}$ load. The highest significant pressure reduction ( $\mathrm{p}<$ 0.05 ) was $6.81 \%$, which was recorded at the posterior proximal subregion during the posterior air bladder inflation for the $50 \mathrm{~kg}$ load. Furthermore, the mean pressure at the posterior proximal was significantly higher than the posterior middle and distal during both inflation and deflation (Table 1). This result was also similar to the achievement of Al-Fakih, Arifin [19], which identified high pressure at the posterior proximal than middle and distal during a stance phase. However, in the current study, the inflation of posterior air bladder had minimized pressure at the posterior proximal. Therefore, this paper suggests that pressure can be reallocated and controlled at the posterior subregions to avoid discomfort and pain at the posterior side during daily activities.

Moreover, the mean pressures at lateral side were recorded as $34.36 \mathrm{kPa}(\mathrm{p}<0.05, \mathrm{SD}=0.23)$, and $42.96 \mathrm{kPa}(\mathrm{p}<0.05, \mathrm{SD}=0.20)$ during the deflation of lateral air bladder for both $50 \mathrm{~kg}$ and $65 \mathrm{~kg}$ loads, respectively. However, the mean pressures at the lateral side were recorded as $36.81 \mathrm{kPa}(p<0.05, S D=$ $1.13)$, and $46 \mathrm{kPa}(\mathrm{p}<0.05, \mathrm{SD}=1.6)$ during lateral air bladder inflation for the $50 \mathrm{~kg}$ and $65 \mathrm{~kg}$ loads, respectively (Fig. 1). In the lateral distal, the pressure was significantly higher during inflation than deflation of lateral air bladder for the $50 \mathrm{~kg}$ and $65 \mathrm{~kg}$ loads (Table 1). This increase in pressure was consistent with a study conducted by Dumbleton, Buis [26], which observed a higher pressure at the lateral distal. Thereby, the current study recommended that the user can minimize the high pressure at the fibula head in the lateral side by increasing pressure at the lateral distal through inflation of the lateral air bladder and reallocating pressure distribution.

This study identified that there was no increasing or decreasing in pressure at all medial subregions for both loads during the inflation and deflation medial air bladder. Except for the medial distal of $50 \mathrm{~kg}$ load and medial middle of $65 \mathrm{~kg}$ load, the pressure was slightly and significantly reduced by $2.25 \%$ at medial distal, and $3.44 \%$ (but not significantly) at the medial middle during the inflation of the medial air bladder (Table 1). The result suggested that the pressure management system could fix the pressure in the soft tissues and muscles and minimize other subregions of the medial side simultaneously.

There was no statistical difference in pressure at the anterior side during inflation of the lateral and medial air bladders. The pressure was reduced at the anterior side as compared to the result of a study conducted by Ali, Osman [21], which found that the greatest value of pressure was observed at the anterior $(84.9 \mathrm{KPa})$ for the seal-in X5 liner and $(60.2 \mathrm{kPa})$ for the Dermo liner during a walk. Moreover, 
Eshraghi, Osman [22] identified that the mean peak pressure of the magnetic system was $79.26 \mathrm{kPa}$ at the anterior side. However, in the current study, the highest value of pressure during the stance phase recorded at the anterior side was $55.89 \mathrm{kPa}$. Therefore, this study suggested that a pressure management system with a 3D printed socket could provide comfort for the user and reduce patellar tendon injury due to low pressure in the anterior side as compared to other suspension systems. Moreover, the pressure at the anterior middle was greater than the pressure at the anterior proximal and distal, except for pressure at the anterior distal for $65 \mathrm{~kg}$ load. This finding corresponded to the study conducted by Al-Fakih, Arifin [19], which noticed that pressure at the anterior proximal and distal was less than the anterior middle for the artificial stump during the stance phase.

The findings of this work were limited because the interface pressure measurement was conducted only by using the universal tensile machine with the static load. Therefore, further human subjects are needed to identify the interface pressure of the stump by using a pressure measurement system during locomotion.

\section{Conclusion}

In this study, a 3D printed prosthetic socket with tiny air bladders which was integrated with a liner was constructed. The inflation and deflation of those air bladders were controlled by using a pressure management system that was attached to the prosthesis pylon. The pressure management system has capability to deal with loose or gap inside the prosthetic socket and redistribute interface pressure. Thereby, this system could enhance the fitting of a stump within the prosthetic socket and reduce the occurrence of skin injury. Moreover, the user could deflate the air bladders to minimize interface pressure at boney areas and inflate air bladder to increase the attachment of muscle and soft tissues. The interface pressure was reduced at the posterior, medial region, patellar tendon, and fibula head by using the pressure management system. Therefore, it can be concluded that the inflation of air bladders can minimize fractions and high pressure at the subregions of the stump, and the user could rearrange pressure distribution.

\section{Methodology}

\section{D Printed Socket Integrated with air bladders}

The acquisition of an amputee's residual limb shape and size was taken by using a 3D laser scanner that was available at the Centre for Biomedical and Technology Integration (CBMTI) Laboratory in the University of Malaya. Then, the computer-aided design (CAD) and SolidWorks program were employed to enhance the socket design and determine the size and dimension of external adaptors of the socket. Actually, the acquired shape was used to design the 3D model prosthetic socket which accommodates the pressure and volume management systems. The final 3D socket design was directly 3D-printed by using a Stratasys Connex 500 printer, as shown in Fig. 2. This 3D printed socket was built with tiny air bladders that were integrated with the socket liner. This 3D socket has three air bladders located at the 
posterior, lateral, and medial sides. Vero White material was used for the outer layer and Tango Black for the inner layer of the socket. The inner layer was in contact with an artificial residual limb, and it is a replacement for the expensive silicon liner. This artificial residual limb was fabricated from silicon material with the same amputee's stump shape and dimension of the amputee stump with consideration that the tibia, fibula bones, and muscles have soft tissue regions.

\section{An interface Pressure Management System Development}

The pressure management system included an electronic device which was connected with air bladders that were integrated within the 3D printed socket, through tiny pipes. The electronic device consisted of four main parts, which included pumps, valves, keypad, and Arduino Board. The major function of the electronic system was to inflate and deflate the air bladders to control the desired pressure inside the 3D printed socket by using the preprogramed keypad. The Arduino control unit kick-started the whole system. This electronic system was attached to the pylon of the prosthetic device in a small box, and the user can press on the keypad to run pumps for inflation and valves for deflation air bladders for each side separately (Fig. 3).

This 3D printed socket had three air bladders which were connected to three separate subsystems, including mini pumps and valves. The first subsystem was a bladder located on the posterior aspect of the socket and connected to a pump and a release valve to inflate/deflate the posterior air bladder to reduce pressure at the posterior region. The two bladders were placed on lateral and medial sides and separately connected to one pump and one release valve. These two bladders were inflated and deflated simultaneously, and thus lift up the artificial stump to reduce the force applied into the crest tibia, medial side, and patellar tendons. The air bladders were connected with tiny air tubes through the 3D printed external adaptors located on the socket wall, and on the other end, they were connected to the pumps and release valves.

\section{Universal Tensile Machine Experiment (UTM)}

Universal Tensile Machine (Instron3369) at the Mechanical Laboratory in University of Malaya was used to apply $485 \mathrm{~N}$ and $635 \mathrm{~N}$ loadings into the prosthetic device in normal static standing to mimic the human body weight, as illustrated in Fig. 5 . The artificial stump, which was made from silicone material, was $1.5 \mathrm{~kg}$. Thereby, the total weight applied to prosthesis was $50 \mathrm{~kg}$ and $65 \mathrm{~kg}$. The universal tensile machine compressed the prosthetic device by applying a static load. The compression started from a low load, and then reached the desired value. The load was held on for the time setting, as shown in Fig. 4. The universal tensile machine was used according to the ISO 10328 protocols, which are International Standards for Structural Testing of Lower-Limb Prostheses.

\section{Tekscan System Pressure Mapping with UTM}


In this study, four transducers were used to determine the interface pressure (F-Socket Transducers, $9811 \mathrm{E}$ Type) reduction. The transducers were located inside the socket at the anterior, posterior, lateral, and medial sides. These transducers were calibrated and equilibrated through an air compressor with $100 \mathrm{kPa}$ pressure at the Motion Analysis Laboratory in University of Malaya. It was in accordance with the manufacturer's instructions before conducting the pressure evaluation[27]. The pressure profiles were recorded by using Tekscan software Version 6.51. Each transducer array was affixed to the anterior, posterior, medial, and lateral of the 3D printed socket. Adhesive spray (3M Spray Mount Adhesive) was employed to fix the transducers at the inner wall sides of the socket to cover all areas and avoid displacement. Block angle platform ( $20^{\circ}$ slope) was used with heel and forefoot in static loading during the test to mimic heel strike and toe-off during the normal stance phase, as illustrated in Fig. 5 . The pressure interface was evaluated during static loading of inflating and deflating air bladders inside the 3D printed socket.

The pressure was recorded during inflation and deflation of air bladders at all sides of the artificial stump. For an acquisition parameter in the Tekscan system, pressure measurement acquisition was performed for 12 seconds with a sample rate of $50 \mathrm{~Hz}$ in two different phases. Firstly, a static phase was to deflate air bladder for 6 seconds; secondly, a dynamic phase was to inflate air bladder for 6 seconds for each side, separately. A total of 90 trials were carried out to determine the interface pressure. Therefore, the pressure was recorded three times for each side of the stump, except for the anterior side, which had six trails to inflate or deflate the lateral and medial air bladders for $50 \mathrm{~kg}$ and $65 \mathrm{~kg}$ loads with three activities, including heel strike, foot flat, and toe-off.

\section{Data analysis}

The mean pressures for every three trials of posterior, lateral and medial regions and six trails for the anterior region were extracted for each heel strike, foot flat, and toe-off, separately. Then, average pressure was taken for heel strike, foot flat and toe-off in the form of stance phase for mean pressure that was gained for each region, for purposes of statistical analyses through MATLAB R2019b software. Data statistical analysis in the form of t-test to compare pressure values and the standard deviation (SD) was applied to the sampling data extracted from the stance phase during inflation and deflation of air bladders.

\section{Abbreviation}

Not Applicable

\section{Declarations}

\section{Ethics approval and consent to participate :}

no clinical trial in this study 


\section{Consent for publication:}

Not applicable

\section{Competing interests:}

The authors declare that no competing financial interests in this study.

\section{Availability of data and material:}

The experimental data used to support the results of this research are included within the article.

\section{Funding:-}

University of Malaya internal grant (project no: BK101-2016).

\section{Authors' contributions}

- MAS designed the pressure control system as portable kit attached in the pylon and the protocol, evaluated the initiation test of system and F- Socket transducers calibration, conducted theexperiments to measure pressure reduction, carried out the mechanical testing, collected and analyzed the data, discussed the results and drafted and finalized the

- NA supervised the overall project and assisted in revising the manuscript to give comment corrections, fabricated the second artificial stump, helped during the test.

- EA co-supervisor of project, developed 3D printed prosthetic socket with integrated air bladders, helped in revising the manuscript, fabricated the first artificial stump.

- All the authors read and commented on the manuscript.

\section{Acknowledgments}

This study was supported by University of Malaya internal grant (project no: BK101-2016). This support covered materials needed for 3D printed and prosthetic and control system components. The authors express their utmost gratitude and thankfulness for Hadramout Establishment for Human Development, and highly appreciate the cooperation and kind assistance of Eng. Mouaz Al Kouzbary.

\section{References}

1. WHO, Global report on diabetes: executive summary. 2016, World Health Organization. 
2. Wolf $\mathrm{SI}$, et al. Pressure characteristics at the stump/socket interface in transtibial amputees using an adaptive prosthetic foot. Clin Biomech. 2009;24(10):860-5.

3. Al-Fakih E, Abu Osman N, Mahmad F, Adikan. Techniques for interface stress measurements within prosthetic sockets of transtibial amputees: A review of the past 50 years of research. Sensors. 2016;16(7):1119.

4. Laing S, Lee PV, Goh JC. Engineering a trans-tibial prosthetic socket for the lower limb amputee. Annals of the Academy of Medicine-Singapore. 2011;40(5):252.

5. Mai A, et al. Effect of prosthetic foot on residuum-socket interface pressure and gait characteristics in an otherwise healthy man with transtibial osteomyoplastic amputation. JPO: Journal of Prosthetics Orthotics. 2012;24(4):211-20.

6. Mak AF, Zhang M, Boone DA. State-of-the-art research in lower-limb prosthetic biomechanics-socket interface: a review. J Rehabil Res Dev. 2001;38(2):161-74.

7. Paternò $L$, et al. Sockets for limb prostheses: a review of existing technologies and open challenges. IEEE Trans Biomed Eng. 2018;65(9):1996-2010.

8. Saeed Zahedi O. FIMechE, Research in Lower Limb Prosthetics, in Atlas of amputations and limb deficiencies: surgical, prosthetic, and rehabilitation principles, J.W. Michael and J.H. Bowker, Editors. 2004, American Academy of Orthopaedic Surgeons Rosemont, IL.

9. Mehmood W, et al., Comparative study of the circumferential and volumetric analysis between conventional casting and three-dimensional scanning methods for transtibial socket: A preliminary study. Proceedings of the Institution of Mechanical Engineers, Part $\mathrm{H}$ : Journal of Engineering in Medicine, 2019. 233(2): p. 181-192.

10. Herr H, Wilkenfeld A, Blaya J. Patient-adaptive prosthetic and orthotic leg systems. in Proceedings of the 12th Nordic Baltic Conference on Biomedical Engineering and Medical Physics. 2002.

11. Sengeh $\mathrm{DM}$, Herr $\mathrm{H}$. A variable-impedance prosthetic socket for a transtibial amputee designed from magnetic resonance imaging data. JPO: Journal of Prosthetics Orthotics. 2013;25(3):129-37.

12. Gholizadeh, et al. Transtibial prosthetic socket pistoning: Static evaluation of Seal-In $\circledast X 5$ and Dermo® Liner using motion analysis system. Clin Biomech. 2012;27(1):34-9.

13. Baars E, Geertzen J. Literature review of the possible advantages of silicon liner socket use in transtibial prostheses. Prosthet Orthot Int. 2005;29(1):27-37.

14. Beil TL, Street GM. Comparison of interface pressures with pin and suction suspension systems. Journal of Rehabilitation Research \& Development, 2004. 41(6).

15. Eshraghi A, et al. Development and evaluation of new coupling system for lower limb prostheses with acoustic alarm system. Scientific reports. 2013;3:2270.

16. Gholizadeh $\mathrm{H}$, et al. Transtibial prosthesis suspension systems: systematic review of literature. Clinical biomechanics. 2014;29(1):87-97.

17. Street G. Vacuum suspension and its effects on the limb. Orthopadie Technik. 2006;4(1):7. 
18. Dillingham TR, et al. Use and satisfaction with prosthetic devices among persons with trauma-related amputations: a long-term outcome study. Am J Phys Med Rehabil. 2001;80(8):563-71.

19. Al-Fakih EA, et al. Optical fiber Bragg grating-instrumented silicone liner for interface pressure measurement within prosthetic sockets of lower-limb amputees. Journal of biomedical optics. 2017;22(8):087001.

20. Gholizadeh, et al. Clinical implication of interface pressure for a new prosthetic suspension system. Biomedical engineering online. 2014;13(1):89.

21. Ali S, et al. Clinical investigation of the interface pressure in the trans-tibial socket with Dermo and Seal-In X5 liner during walking and their effect on patient satisfaction. Clin Biomech. 2012;27(9):943-8.

22. Eshraghi $A$, et al. An experimental study of the interface pressure profile during level walking of a new suspension system for lower limb amputees. Clin Biomech. 2013;28(1):55-60.

23. Pirouzi $G$, et al. Development of an air pneumatic suspension system for transtibial prostheses. Sensors. 2014;14(9):16754-65.

24. Goswami J, et al. Walking in a vacuum-assisted socket shifts the stump fluid balance. Prosthet Orthot Int. 2003;27(2):107-13.

25. Gailey R, et al., Review of secondary physical conditions associated with lower-limb amputation and long-term prosthesis use. Journal of Rehabilitation Research \& Development, 2008. 45(1).

26. Dumbleton T, et al., Dynamic interface pressure distributions of two transtibial prosthetic socket concepts. Journal of Rehabilitation Research \& Development, 2009. 46(3).

27. Buis A, Convery P. Calibration problems encountered while monitoring stump/socket interface pressures with force sensing resistors: techniques adopted to minimise inaccuracies. Prosthet Orthot Int. 1997;21(3):179-82.

\section{Figures}

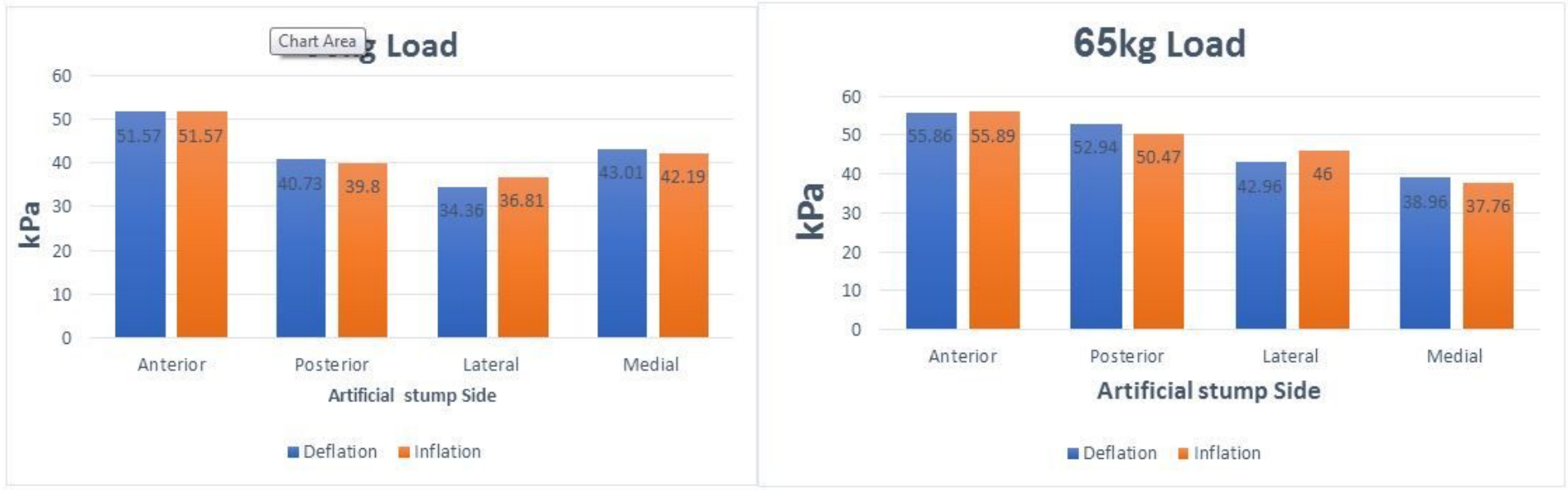

Figure 1 
Mean pressure for the four main regions of the artificial stump during a stance phase

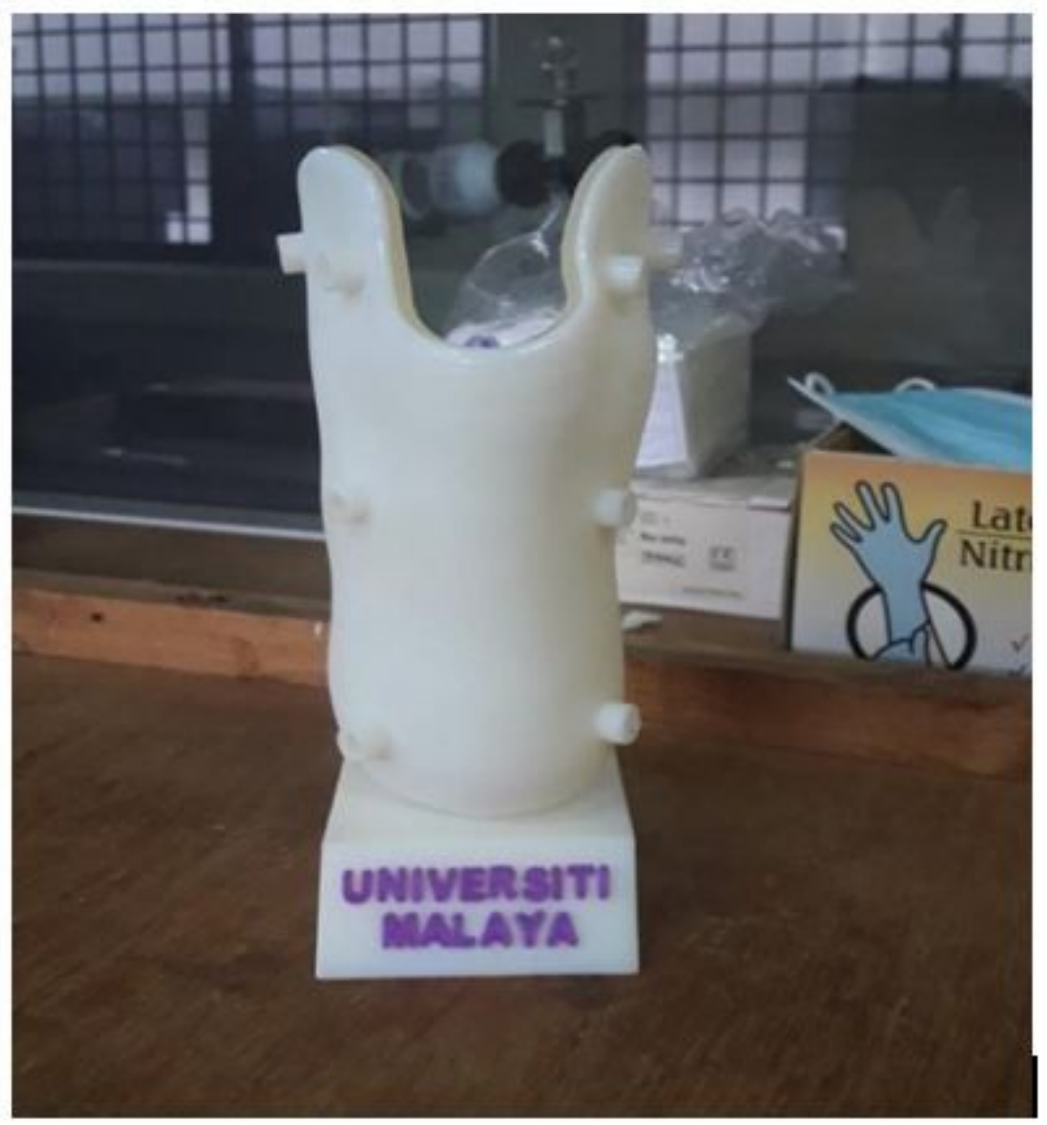

Figure 2

Final Design of 3D Printed Socket

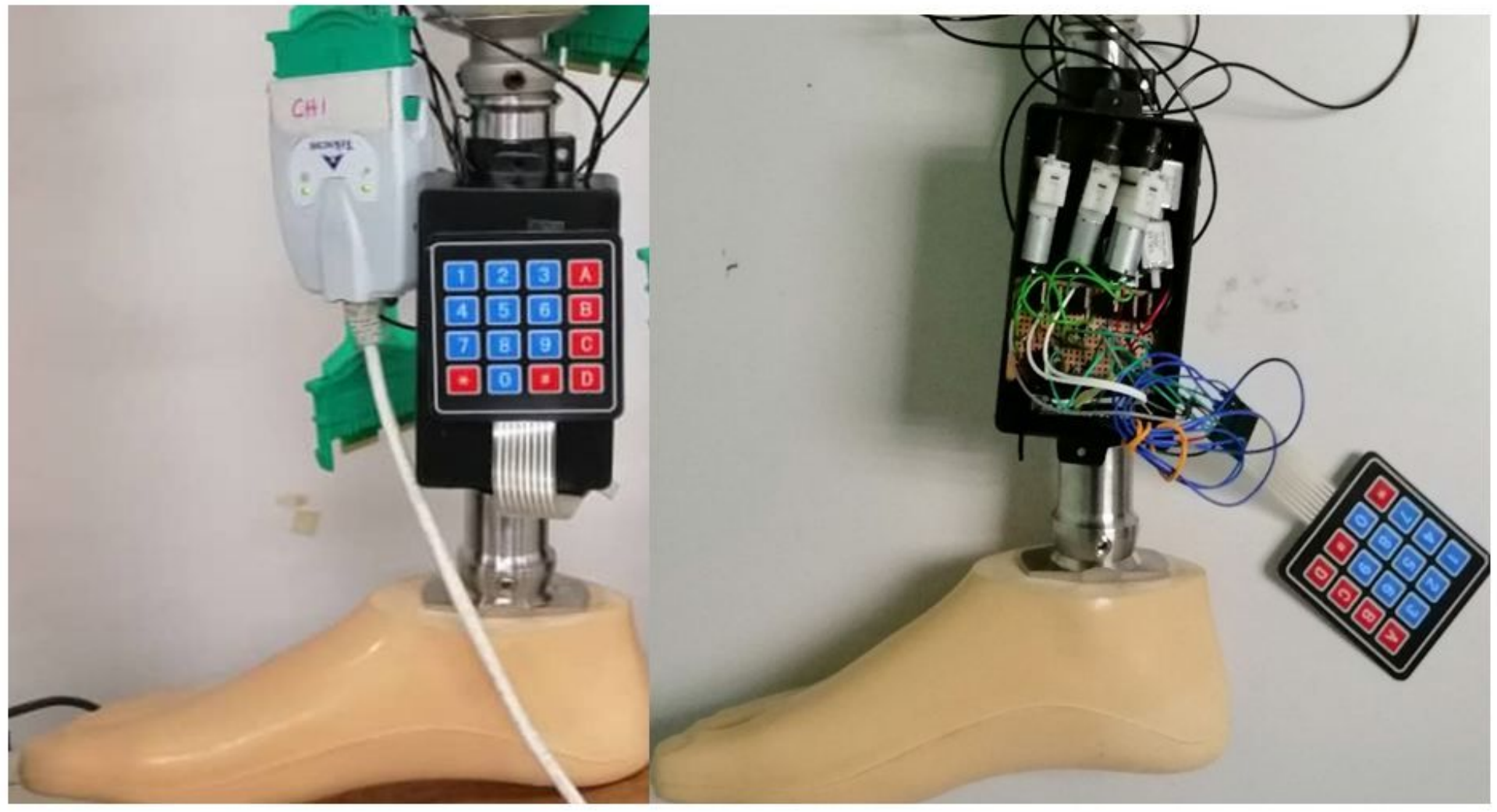


Figure 3

Electronic system with prosthesis pylon
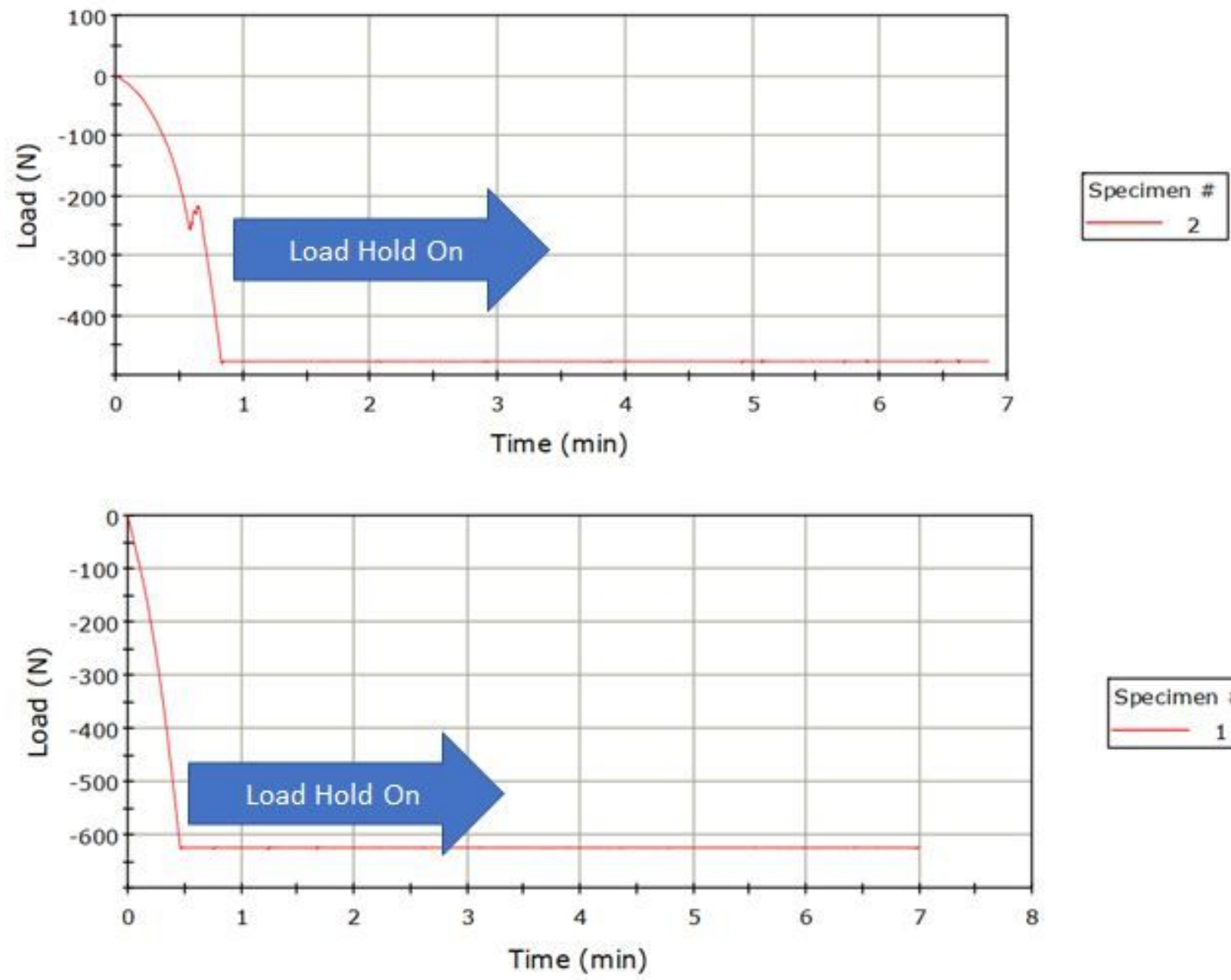

Specimen \# 1

Figure 4

Compression loads for 485 and $635 \mathrm{~N}$ through a universal tensile machine (Instron3369) into a prosthetic device 

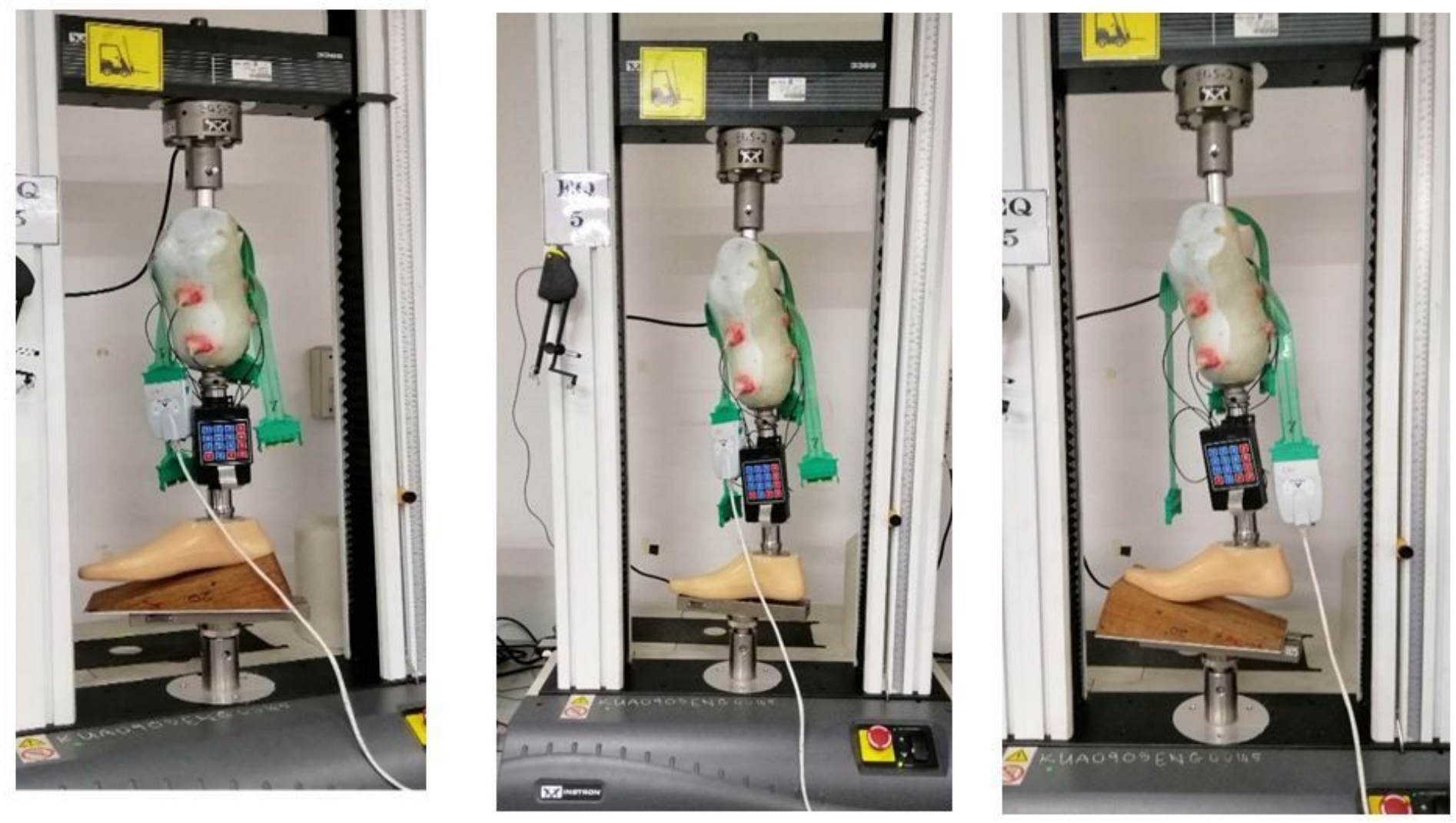

Figure 5

Vertical compression load $485 \mathrm{~N}$ and $635 \mathrm{~N}$ transferred a bending load to resemble human body weight during heel strike, foot flat, and toe-off 